\title{
Clinical and Epidemic Characteristics of Patients with Tuberculosis in Early and Preschool Children
}

\author{
Nadezhda Gulyaeva ${ }^{1,}$, Maria Vinokurova $^{2}$, Olga Ivanova $^{1}$ and Valentina Adamova ${ }^{1}$ \\ ${ }^{I}$ North-Eastern federal university named after M.K. Ammosov, Medical institute, 27, Oyunsky St., Yakutsk, 677000, \\ Republic of Sakha (Yakutia), Russia \\ ${ }^{2}$ State budgetary institution of the Republic of Sakha (Yakutia) scientific and practical center "Phthisiatry", \\ 93, P. Alekseeva St., Yakutsk, 677000, Republic of Sakha (Yakutia), Russia \\ "Corresponding author. Email: gulyaeva.nv@s-vfu.ru
}

\begin{abstract}
The aim of the research is to study modern clinical and epidemic characteristics of tuberculosis patients of early and preschool children in the Republic of Sakha (Yakutia). Traditional methods of assessing the patient's clinical condition and history have been used. Analysis of radiological and laboratory studies was carried out. Patients were divided into 2 groups: the 1st group was made up of 120 patients $(70.6 \%)$ from established contact with bacteriodilators, the $2 \mathrm{~d}$ group included 50 tuberculosis patients of children $(29.4 \%)$ from unknown contact. A study of the structure of tuberculosis complications showed that children of the first group had complications in 26 cases (21.7\%), obstruction of pulmonary tissue was diagnosed in 16 people $(13.3 \%)$, bronchopulmonary lesion -8 children $(6.7 \%)$, pulmonary tissue atelectasis $-7(5.8 \%)$, pleurisy $-4(3.3 \%)$, decay $-3(2.5 \%)$, polysitis in one $(0.8)$. In the second group of patients, complications were diagnosed less often and established in 6 cases (12\%).
\end{abstract}

Keywords: tuberculosis, preschool children, clinical and epidemic characteristics

\section{INTRODUCTION}

Tuberculosis is a widespread infectious disease in the world. According to WHO, 10 million new cases of active tuberculosis are diagnosed annually in the world, approximately $10 \%$ are children under the age of 15 , which leads to about 80 thousand deaths [1]. Currently, the Russian Federation is experiencing a stabilization of the epidemic situation with a tendency to improve tuberculosis, as evidenced by the dynamics of the main indicators on tuberculosis. For 10 years (from 2008 to 2017), the following main indicators for tuberculosis decreased: tuberculosis incidence - from 85.1 to 48.3 per 100 thousand population (by $43.2 \%$ ); tuberculosis incidence among children aged $0-14$ years - from 15.3 to 9.7 per 100,000 children (36.6\%); the prevalence of tuberculosis at the end of the year - from 190.7 to 109.8 per 100 thousand population (by $42.4 \%$ ) ; bacterization in patients with tuberculosis from 80.3 to 46.0 per 100 thousand population (by
$42.7 \%$ ); tuberculosis mortality - from 17.9 to 6.5 per 100 thousand population (by $63.7 \%$ ) [2].

The decisive factor determining the occurrence of tuberculosis in children is their cohabitation with the bacteriospecifier in the focus of tuberculosis infection. In children aged $0-14$ years, direct data on the presence of drug-resistant tuberculosis (DR) of the causative agent are generally absent, since the proportion of tuberculosis with bacterization is stably small (5-6\%). The gradual improvement of the epidemic situation of tuberculosis in the country determines the feasibility of moving from mass single-type anti-tuberculosis measures to targeted ones in risk groups, primarily in tuberculosis centers, where the incidence of children and adolescents is $25-35$ times, and adults 2-23 times higher than that among the population [3-5].

In tuberculosis foci, a child acquires a disease caused by mycobacteria of tuberculosis (MTB), obtained from the source of infection, with a known 
spectrum (DR), which serves as a reference when prescribing chemotherapy (CT) to children [6-8].

In the Republic of Sakha (Yakutia), the incidence of tuberculosis in children aged 0-14 years old remains higher than in the Russian Federation. In 2017 amounted to 17.3 per 100 thousand people, in 2018 . amounted to 15.0 per 100 thousand of the population, which is also higher than the indicators for the Russian Federation. The incidence rate of adolescents in 2017 was 57.1 , in $2018-40.5$, which is almost 2 times higher than the all-Russian indicators. Meanwhile, in recent years, the republic has a high frequency of drug resistance

M. tuberculosis, including poly- and multidrug resistance (MDR MTB) [9]. It is generally known that the incidence rate tuberculosis of children clearly reflects the epidemic situation in the region. Regional features of the republic (low population density, extreme climate, long distances between settlements) have always complicated the process of organizing the diagnosis of tuberculosis in children.

In recent years, the diagnostic methods have been replenished with X-ray computed tomography (RCT), which positively affects quality of tuberculosis diagnosis in children and immunodiagnostics methods allowing to identify groups with high risk of developing the disease [10-12].

According to "Phthisiology" depatment the territorial incidence rate (form No. 8, taking into account departments) in the period 2015-2018 tended to decrease from 69.7 to 54.2 per 100 thousand population. In 2018 , compared to 2015 , it decreased by $22.2 \%$ [4]. In 2018, the incidence rate of tuberculosis for children in the Russian Federation at the age of $0-14$ years old amounted to 8.3 per 100 thousand children. In the Republic of Sakha (Yakutia), this indicator was recorded significantly higher than the national average, in 2018 it amounted to 15.0 per 100 thousand children, which is 1.8 times higher than the indicators for the Russian Federation [9].

The aim of the study is the modern clinical and epidemic characteristics of tuberculosis patients of early and preschool children in the Republic of Sakha (Yakutia)

\section{METHODS AND MATERIALS}

The study of the clinical course of tuberculosis in children was carried out by examining of 170 patients aged from 0 to 6 years old who were in hospital in specialized establishments. Traditional methods for assessing the clinical condition of the patient and history were used: complaints, epidemic case history - contact of the child with an adult TB patient, information about BCG vaccination, diseases suffered, and social factors were also taken into account. An objective examination determined the nature of local vaccination signs, the response of peripheral lymph nodes, as well as clinical manifestations of the disease. Analysis of radiological and laboratory studies was carried out. All the abovementioned methods of examination were carried out on admission to the hospital and discharge. The examined 170 patients were divided into 2 groups: the 1 st group amounted to $120(70.6 \%)$ patients from established contact with bacteriodilators, the $2 \mathrm{~d}$ group included 50 (29.4\%) tuberculosis patients of children from unknown contact. It should be noted that with an active tuberculosis process in the first group of dispensary records consisted of 116 children $(97 \%)$ of the first group and 41 patients (82\%) of the second group, the remaining children were observed in the third group of records. Data analysis was carried out by Statistica for Windows Version 6. Statistical data processing included finding the mean, mean errors, and estimating intergroup differences by Student's criterion. Differences at $p<0.05$ were considered significant.

\section{RESULTS AND DISCUSSION}

The distribution of patients by age, place of residence and gender showed that in the first group of children aged from 1 to 3 years old there were 65 people $(54.2 \%)$, in the second group there were children from 2 to 4 years old -33 cases $(66 \%)$. In the 1 st group 78 of patients $(65 \%)$ lived in rural areas, in the $2 \mathrm{~d}$ group 32 children $(64 \%)$ lived in urban conditions. The proportion of boys in both groups was higher than that of girls in groups - $65(54.16 \%)$ and $30(60 \%)$, respectively. When studying social factors, it was found that in both groups, children from a full family made up the majority, $95(79.2 \%)$ and $37(74 \%)$, respectively. 75 children $(62.5 \%)$ from the 1 st group and 23 children (46\%) from the $2 \mathrm{~d}$ group lived in houses without improvement.

When studying BCG vaccination coverage and the value of the vaccination mark in tuberculosis patients, children in the trial groups showed some differences. In group 1, out of 120 children, $3(2.5 \%)$ were not vaccinated, the absence of a post-vaccine mark was noted in $13(10.8 \%)$. The value of the "scar" with a size of 4-5-6 mm was found in 68 children $(56.6 \%)$, with a vaccination mark with a size of 1 to $3 \mathrm{~mm}$ was 23 $(19.2 \%)$. In group 2, all 50 patients were vaccinated, the absence of a post-vaccine mark was observed in 2 children $(4 \%)$, the vaccination mark was $2-3 \mathrm{~mm}$ in 11 $(22 \%)$

It should be noted that among children with tuberculosis who were not vaccinated with BCG, all of them were from foci of tuberculosis infection. The scar was $4-5-6 \mathrm{~mm}$ in 30 patients $(60 \%)$. Therefore, there were more persons with no and failure of the vaccination mark after BCG vaccination in the 1 st group 
- 36 children (30\%), 3 children $(2.5 \%)$ were not vaccinated. In the second group, everything was vaccinated, the absence and failure of the "scar" was encountered with approximately the same frequency. This indicates that the magnitude of the vaccination mark does not play a significant role in preventing the disease of tuberculosis of the child.

When considering clinical forms of local tuberculosis in children, it was revealed that tuberculosis of intrathoracic lymph nodes was most often diagnosed, both in the first and second groups.

The greatest importance in the development of the disease is the contact with a tuberculosis patient. This is clearly demonstrated by patients of the 1st group (from foci of tuberculosis infection), where the majority were vaccinated with BCG among sick children and only 3 $(2.5 \%)$ of patients were not vaccinated. It was of interest to study the age of infection with mycobacteria of tuberculosis of children before detecting their tuberculosis disease. In the follow-up groups, a study on the dynamics of tuberculosis samples and the time of diagnosis of tuberculosis showed that the detection of tuberculosis in a child coincided with the establishment of superelevation of tuberculosis sensitivity 3 times more often in sick children from the 1st group living in the foci of infection, but this was noted only in 42 children $(35 \%)$, and in the $2 \mathrm{~d}$ group $-7(14 \%)$. In the remaining patients of group 1 , the duration of MTB infection within 1-3 years before the diagnostic of the disease was 51 people (42.2\%). Among patients of group 2, the same duration of infection (1-3 years) was noted, also in 28 children $(56 \%)$.

We also evaluated data on the preventive chemotherapy for contact with an adult TB patient, superelevation of tuberculosis samples and hyperergic sensitivity to tuberculosis. A detailed analysis of the chemoprevention carried out and the place of its implementation revealed that among patients from the foci of tuberculosis infection it was carried out equally, both in outpatient and inpatient conditions: 33 (27.5\%) and $35(29.2 \%)$, respectively. In group 2, chemoprevention in sanatorium conditions was carried out in 7 cases $(14 \%)$, in outpatient conditions in 15 cases $(30 \%)$. The number of patients treated in the sanatorium is almost 2 times more in the 1st group than in second one, $33(27.5 \%)$ and 7 (14\%), respectively. However, despite the controlled chemoprevention, children, both from the foci of tuberculosis infection and from undisclosed contact, fell ill with tuberculosis. Thus, the risk factors for the development of the disease were, first of all, contact with a tuberculosis patient - a bacteriodilator and the duration of infection with MTB lasting from 1 to 3 years. Children from the foci of tuberculosis infection received preventive chemotherapy both outpatiently and in the sanatorium, which was obviously associated with a number of causes, such as ongoing or new contact with the MTB bacteriodetector, paediatricians' underestimation of the child's clinical condition in comorbidities, and parents' low anxiety about the consequences of infection.

We studied the types of contacts of children from the $1^{\text {st }}$ trial group. The mother had contact with a TB patient in 41 children (34.2\%), with a sick father - 32 $(26.7 \%)$, with a grandfather - $17(14.2 \%)$, with an uncle - $21(17.5 \%)$. Double and triple contact was observed in 27 children $(22.5 \%), 14$ sick children $(11.6 \%)$ were identified from the centers of death. In the diagnosis of adult tuberculosis patients who were in contact with children, infiltrative pulmonary tuberculosis prevailed $41.7 \%$ (50 patients), focal pulmonary tuberculosis $-17(14.2 \%)$, disseminated pulmonary tuberculosis occurred in 7 (5.8 \%), fibrous cavernous - in $6(5 \%)$, extrapulmonary forms of tuberculosis were detected in $2(1.7 \%)$. In 38 adults $(31.6 \%)$, the diagnosis was not indicated in the child's case histories. When studying contacts among adults, we took into account the drug sensitivity of tuberculosis mycobacteria isolated by patients. In 16 patients $(13.3 \%)$, contact was recorded with an adult patient with drug resistance of MTB to PTP, to one drug isoniazid $(\mathrm{H})$ in $3(18.7 \%), 10(62.5 \%)$ showed drug resistance to three drugs, isoniazid, rifampicin and streptomycin $(\mathrm{H}, \mathrm{R}, \mathrm{S})$, to four in 1 patient $(6.3 \%)-$ isoniazid, rifampicin, streptomycin and PASA $(\mathrm{H}, \mathrm{R}, \mathrm{S}$, PAS), to five in 2 (12.5) - isoniazid, rifampicin, streptomycin, PASA and ofloxacin (H, R, S, PAS, Ofx). Consequently, in children of early and preschool age, contact with the mother is most frequent, in second place - with the father, in third - with close relatives. Quite often there were double and triple contacts, common forms of tuberculosis with destructive changes in the lungs and foci of death from tuberculosis, the most dangerous for children living there. Thus, the determining factor in the occurrence of the disease is the massive infection and the duration of contact with the patient with tuberculosis.

The course and outcome of the disease depends on the timely disgnostic of tuberculosis in children. Analysis of diagnostic pathways in children from surveillance groups showed that in the 1st group tuberculosis was detected in $61(50.8 \%)$ children during immunodiagnostics. $25(20.8 \%)$ children of them were identified with a hyperergic sample, $16(13.3 \%)$ - the superelevation of tuberculosis samples, 13 (10.8\%) the monotonicity, $7(5.9 \%)$ - the increase in tuberculosis samples. $34(28.4 \%)$ children were identified During the contact examination, and 25 $(20.8 \%)$ children were identified at doctor's visit. 44 (88\%) patients were identified by immunodiagnosis in group 2, the monotonicity of tuberculosis samples -19 $(38 \%)$ of patients and hyperergic reaction $-12(24 \%)$, on increase of sensitivity to tuberculosis - $9(18 \%)$, superelevation $-4(8 \%)$. It is noted that children of the 
1st group turned to the dispensary for medical care almost 2 times more often than children from an unidentified contact (20.8\% and $12 \%$, respectively), indicating a leading role of the massiveness and duration of infection in the development of clinical signs of the disease.

It is of interest to study tuberculosis sensitivity by Mantu sample with 2 TE in the compared groups of patients.

The average Mantu sample infiltrate size with 2 TE is larger in patients living in the established focus of infection. The frequency of negative and amphibolous reactions to the Mantu sample with $2 \mathrm{TE}$ in the observation groups is approximately the same: 2.5 in the 1 st group and $4.0 \%$ and 2.5 and $4.0 \%$ in the $2 \mathrm{~d}$ group, respectively. Low-positive results (infiltrate size within 5-11 mm) were 2 times more common (44.0 and $21.6 \%$ ) in patients of group 2, i.e., from undetected contact. With the same frequency, expressed responses to tuberculosis were recorded in a dose of $2 \mathrm{TE}$ with intracutaneous administration with infiltrate $12-16 \mathrm{~mm}$ (39.2 and $40.0 \%$ ). However, the frequency of hyperergic tuberculosis sensitivity in tuberculosis patients from the centers of infection was 3 times higher, respectively 34.2 and $10.0 \%$. This confirms the diagnostic and epidemic significance of hyperergic responses, developing in most cases in the presence of prolonged superinfection, leading to an activation of tuberculosis infection in a sick child. At the same time, it should be noted that under the condition of contact with a patient with tuberculosis in most sick children, the sensitivity to tuberculosis was normergic. When discharged from the hospital after a treatment course for a period of 5-6-8 months, the sensitivity to tuberculosis decreased in patients of both groups and the average size of the papule was in the 1 st group $9.3 \pm 0.3 \mathrm{~mm}$, in the $2 \mathrm{~d}$ group $-8.9 \pm 0.5$.

Analysis of clinical manifestations of tuberculosis infection in patients of groups 1 and 2 at the stage of inpatient treatment was carried out. First of all, the symptoms of intoxication and their severity at the time of admission to the children's tuberculosis department were noted. $104(61.2 \%)$ children had moderate symptoms of intoxication, the 1st group of patients amounted to $76(63.3 \%)$ people, the $2 \mathrm{~d}$ group -28 (56\%). Pronounced symptoms of intoxication occurred in a third $(33.3 \%)$ of sick children from group 1 living in conditions of tuberculosis contact. Thus, the symptoms of intoxication in more than $90 \%$ of cases occur in patients living in the foci of infection.

A study of the temperature response to tuberculosis infection in sick children upon admission to the hospital revealed that high body temperature was observed 2 times more often in patients from the foci of tuberculosis infection (12.5\% and $6 \%$, respectively).
Among the $1^{\text {st }}$ group patients, $47(27.6 \%)$ children showed paleness of the skin, $37(21.8 \%)$ children had reduced nutrition. A decrease in skin turgor was noted in $33(19.4 \%)$. Polyadenopathy, as a symptom of tuberculosis intoxication, was observed in almost all patients from both groups. The following enlarged groups of peripheral lymph nodes were most often observed in the 1st group: cervical - in $82.5 \%$ cases, axillary $-53.3 \%$, subcellular $-48.3 \%$, inguinal $35 \%$. There was often a combined lesion of 4 and 6 groups of lymph nodes $(30 \%$ and $16.7 \%$, respectively). Their size also varied, 2-3 sizes were most often observed (3-4 $\mathrm{mm}$ and $5 \mathrm{~mm}$ in diameter), in 62.5 and $45 \%$ cases, respectively. Densely elastic peripheral lymph nodes palpated in $47.5 \%$, soft-elastic in $39.2 \%$ of cases in consistency. In $16(13.3 \%)$ children, no changes were detected.

In the 2nd follow-up group, enlarged cervical peripheral lymph nodes were also palpated - in $82 \%$ of cases, axillary - in $56 \%$, subcellular - in $62 \%$, and inguinal - in $24 \%$. Densely elastic prevailed over softelastic, $60 \%$ and $28 \%$, by consistency, respectively. There is also a lesion of 4 and 6 groups of lymph nodes (30\% and $26 \%$ ). By lymph node size, in $74 \%$ of cases, lymph nodes of size $2(3-4 \mathrm{~mm})$ and in $42 \%-1$ size (1-2 $\mathrm{mm})$ were palpated.

The most persistent sign of the disease was a decrease in body weight, especially in children of early and preschool age living in the foci of tuberculosis infection $(11.34 \pm 0.38 \mathrm{~kg})$, compared with patients from undetected contact $(13.3 \pm 0.46 \mathrm{~kg}, \mathrm{p}<0,001)$. It was noted that on the background of specific treatment of tuberculosis, body weight increase in sick children from the foci of infection occurred slowly, which was due to more pronounced and persistent symptoms of intoxication. It was of interest to study the variants of clinical manifestations of the onset of the disease in both groups: asymptomatic, low-asymptomatic, subacute and acute, the presence of complaints was assessed. Acute and subacute onset of the disease was observed more often in children from the foci of infection. In most patients from both groups, there was a low-symptom onset of the disease. The largest number of complaints were made by patients from the 1 st group, among them there were: coughing in $35(20.6 \%)$ patients, weakness $-11(6.5 \%)$, lagging in psychomotor development -10 $(5.9 \%)$, sweating - in $9(5.3 \%)$, shortness of breath was observed in $5(4.2 \%)$ children. Patients from the $2 \mathrm{~d}$ group made fewer complaints: coughing 5 (10 \%), sweating $6(12 \%)$, weakness $2(4 \%)$, poor appetite 2 (4\%), lagging in psychomotor development 2 (4\%), shortness of breath 1 (2\%) of patients. Complaints took place for a long time before the diagnosis of the disease, some of the children received inpatient treatment in the children's departments of district and city hospitals with diagnoses: SARS, bronchitis, pneumonia. 
Physical data in children of group 1 revealed: wired, wet and dry wheezes in $26(21.7 \%)$ children, which was accompanied by stiff breathing of sound in $20(16.7 \%)$ patients, enlarged liver from $1 \mathrm{~cm}$ or more palpated in $32(26.7 \%)$ patients, deafness of heart tones in 4 $(3.3 \%)$ children. In group 2 , severe breathing in children was noted in $6(12 \%)$ patients, wheezing was auditioned in $2(4 \%)$ patients, enlarged liver was palpated in 9 patients, which amounted to $18 \%$ of cases. When studying the data of the general blood test in group 1, lymphocytopenia was observed in 113 patients, which was $94.2 \%$, monocytosis in 105 $(87.5 \%)$, anemia in $88(73.3 \%)$ children, an increase in the rate of red blood cell subsidence (ESR) was observed in $35(29.2 \%)$ patients and reached up to $65 \mathrm{~mm}$ per hour. Leukocytosis occurred in 39 patients, which was $32.5 \%$, eosinophilia was in $22(18.3 \%)$ children. In group 2, lymphocytopenia also occupied a leading place and was observed in $46(86 \%)$ children, monocytosis in 42 (84 \%) people, anemia in $27(54 \%)$ patients, leukocytosis was noted in $19(38 \%)$ patients, eosinophilia was observed in 17 (34\%) children.

More pronounced anaemia was observed in 88 $(73.3 \%)$ children from foci of tuberculosis infection in patients of both groups, the data difference was significantly $\mathrm{t}=2.41, \mathrm{p}<0.01 \mathrm{p}=0.0088$ according to the Student's test. A slight acceleration of red blood cell subsidence was also noted in patients of the first group. All patients from both groups showed lymphocytopenia, eosinophilia and monocytosis, the difference between both groups was not reliable. Thus, analysis of hemograms showed that at the beginning of the development of tuberculosis infection in children, the most characteristic changes were anemia, lymphocytopenia, monocytosis, acceleration of red blood cell subsidence and eosinophilia. It should be emphasized that the change in the "red blood" and the acceleration of red blood cell subsidence was observed in patients of the first group, and the eosinophilia indicator was observed 2 times more often in patients of the second group, which once again confirmed the allergic orientation of tuberculosis infection.

The frequency, multiplicity and mass of bacterization in children was determined according to microbiological studies included in the mandatory complex of examination of patients. The number of bacteriodispecifiers from the 1st group was $8(6.7 \%)$ patients with complicated course of disease and bilateral involvement of intrathoracic lymph nodes from the $2 \mathrm{~d}$ group, and $1(2 \%)$ child was identified. A relevant study was the drug sensitivity of MBT in patients with tuberculosis in children due to the fact that the disease was more common with exogenous superinfection. Resistance to anti-tuberculosis drugs was determined in $3(2.5 \%)$ patients from group 1 . Mono-resistance of MBT to streptomycin was determined in one patient, in the second child resistance to combination of streptomycin and isoniazid. In the third child, multidrug resistance to 4 drugs was determined: isoniazid in combination with rifampicin, streptomycin and ethionamide. In other patients, drug sensitivity was maintained.

A study of the complications structure of tuberculosis showed that the first group complications occurred in $26(21.7 \%)$ cases, obstruction of pulmonary tissue was detected in $16(13.3 \%)$ people, bronchopulmonary lesion $-8 \quad(6.7 \%)$ children, pulmonary tissue atelectasis $-7(5.8 \%)$, pleurisy -4 $(3.3 \%)$, decay $-3(2.5 \%)$, polysitis in one $(0.8)$ At the same time, $3(2.5 \%)$ children had several types of complications at the same time, such as bronchopulmonary lesion and exudative pleurisy in 1 $(0.8 \%)$ child, atelectasis and insemination in $2(1.6 \%)$ children.

In the second group of patients, complications were diagnosed less often and established in $6(12 \%)$ cases. Obstruction of pulmonary tissue was recorded in $6 \%$ of patients ( 3 children), bronchopulmonary lesion $-4 \%$ (in 2 patients), atelectasis $2 \%$ (in 1 child). It should be noted that there were complications of the same type in the second group of children. Therefore, complications, including combined ones, are more common in children who were in contact with an adult patient from the foci of tuberculosis infection.

Some influence on the course of the disease was the presence of comorbidities diagnosed in the hospital. The analysis of comorbidity revealed that patients of group 1 were twice as likely to undergo clay invasions, enterobiosis among them was diagnosed in $24(20 \%)$ and in $6(12 \%)$ children of group 2, intestinal infections were also more common in children from group 1 , dysentery in $25(20.8 \%)$ children compared to $6(12 \%)$ patients of group 2. Patients in group 2 were more likely to have acute respiratory viral diseases, in $40(80 \%)$ children compared to $74(61.7 \%)$ patients in group 1 , tonsillitis occurred in $16(32 \%)$ and 19 (15.8\%) people, respectively. The child successively suffers several infections in the hospital, on the background of primary tuberculosis and if these are acute respiratory viral infections, up to 5 times during the entire period of inpatient treatment of the main disease. When studying this question, we noted that patients of group 1, most often, carry from 2 to 4 infections in the hospital, patients of groups 2, mainly 2-3 infections. This indicates a weakening of the resistance of the body of the patient with tuberculosis, which will make it possible to attach a viral infection that affects the nature of the course of the underlying disease. A more accelerated course was characterized by rapid positive process dynamics in the first 3 months of treatment and was observed in $62(51.6 \%)$ patients of group 1 . The slow course is characterized by a gradual improvement in all indicators (in different terms after 6 months of 
treatment). This disease course occurred in this group of children in $53(44.2 \%)$ cases. The wave-like course of the process was noted only in $5(4.2 \%)$ patients with complicated forms of tuberculosis and were characterized by alternating activation of the process with periods of relative stabilization, and clinically there was a slow quiescence of the process and regression of it, while maintaining moderate sensitivity to tuberculosis. In patients of group 2, accelerated flow was observed in 20 (40\%) patients, delayed in 24 $(48 \%)$, wave-like in $6(12 \%)$ patients. Thus, the frequency of delayed and wave-like flow $(60 \%)$ is higher in patients from unidentified contact, than in children whose source of infection is known (48.4\%). Perhaps this is due to the fact that in the known centers of infection, children used methods of specific prevention of tuberculosis and diagnosis of the disease was more timely. Certain difficulties in treating patients with respiratory tuberculosis were the side effects of anti-tuberculosis drugs, which were directly dependent on the duration of chemotherapy and the presence of aggravating factors.

Adverse reactions were observed equally often in both the first $(55.0 \%)$ and second groups $(54.0 \%)$, toxic hepatitis occurred in $4(3.3 \%)$ patients from the foci of infection. Treatment of the observed tuberculosis patients of children was carried out according to standard chemotherapy regimens, depending on the form of severity of the disease and the presence of complications. At the same time, careful monitoring of the general condition was ensured, taking into account $\mathrm{X}$-ray tomography and laboratory data. The duration of inpatient treatment in children of the 1st group was on average $320.4 \pm 12.7$ bed-days. In patients with undefined contact $-259.4 \pm 19.6$ bed-days, the difference was reliable at $\mathrm{t}=2.62, \mathrm{p}<0.01 \mathrm{p}=0.0052$. This is due to a more severe course of disease in children from foci of tuberculosis infection.

\section{CONCLUSIONS}

Thus, tuberculosis infection in children of early and preschool age is severe in the presence of contact with a diseased bacteriodilator, with a pronounced clinicalradiological picture of primary tuberculosis, with persistent symptoms of intoxication, complicated and torpid course of a specific process. Children with tuberculosis were from foci of tuberculosis infection and they were not previously vaccinated with BCG. The chemoprophylaxis was carried in half of patients from the foci of tuberculosis infection, both in outpatient and inpatient conditions. Insufficient coverage of children with preventive treatment is associated in most cases with a lack of parental commitment to the chemoprevention of tuberculosis infection. One of the main factors in the development of the disease is the late diagnostic, often associated with difficulties in differential diagnosis of tuberculosis with various nonspecific diseases and underestimation of the role of the epidemic factor in contact with tuberculosis patients.

\section{REFERENCES}

[1] World Health Organization. Global tuberculosis report, 2018. Retrieved from: http://apps. who.int/iris/bitstream/handle/10665/274453/97892 41565646-eng.pdf?ua=1 (Accessed Nov. 2020).

[2] O.B. Nechaeva, Epidemic situation of tuberculosis in Russia, Tubercul. and lung dis. 96(8) (2018) 1524. Retrieved from: https://doi.org/10.21292/20751230-2018-96-8-15-24

[3] C.G.M. Erkens, M. Kamforst, I. Abubakar et al., Tuberculosis contact investigation in low prevalence countries: a European consensus, Eur. Respir. J. 3(64) (2010) 925-949.

[4] S. Tornee, J. Kaewkungwal, W. Fungladda et al., The association between environmental factors and tuberculosis infection among household contacts, South. Asian J. Trop. Med. Public Health 36(4) (2005) 221-224.

[5] J.M. Tufariello, J. Chan, J.L. Flynn, Latent tuberculosis mechanisms of nost and bacillus that contribute to persistent infection, Inf. Dis. 3(9) (2003) 578-590.

[6] M.E. Lozovskaya et al., Peculiarities of clinical course and chemotherapy of tuberculosis in children from foci with different spectrum of drug sensitivity of mycobacteria, Tuberc. and lung dis. 97(9) (2019) 22-27. Retrieved from: https://doi.org/10.21292/2075-1230-2019-97-9-2227

[7] A.A. Starshinova, Tuberculosis in children from the family focal point of infection (diagnosis, clinical course and prevention), Ph.D thesis, St. Petersburg Res. Inst. of Phthisiopulmonol., 2013, $46 \mathrm{p}$.

[8] E.A. Tsygankova, Features of tuberculosis in young children and factors contributing to its development in the conditions of pathomorphosis of tuberculosis infection, Ph.D thesis, Central Res. Inst. of Tubercul. RAMS, Moscow, 2012, 22 p.

[9] G.I. Alekseeva et al., Tuberculosis: epidemiology and organization of the modern prevention of the Far North (on the example of the Republic of Sakha (Yakutia), Science, Novosibirsk, 2015.

[10] N.A. Gulyaeva, N.G. Pavlov, Questions of general phthisiology, Yakutsk, 2019. 
[11] N.A. Gulyaeva, V.D. Adamova, Tuberculosis in children in the foci of tuberculosis infection in the Republic of Sakha (Yakutia), Modern probl. of sci. and ed. 4 (2020). Retrieved from: www.scienceeducation.ru/ru/article/view?id=29995

[12] E.F. Luginova, L.P. Shepeleva, O.I. Guryev, Trends of tuberculosis in children in the Republic of Sakha (Yakutia) over a 10-year period on the background of the introduction of new diagnostic technologies, Tuberc. and lung dis. 95(1) (2017) 510. Retrieved from: https://doi.org/10.21292/20751230-2017-95-1-5-10

[13] O.D. Kondratieva, M.N. Kondratyev, S.P. Zorina, Main indicators of anti-tuberculosis activity in the Republic of Sakha (Yakutia): Statistical collection, Yakutsk, 2019.

[14] V.A. Aksenova, L.A. Baryshnikova, N.I. Klevno, Topical issues of screening children for tuberculosis, Tuberc. and lung dis. 6 (2013) 7-8. 